\title{
Valores Sociales y Hábitos Mediáticos en una Muestra de Universitarios
}

\section{Félix Moral Toranzo}

Departamento Psicología Social. Antropología Social, Trabajo Social y Servicios Sociales. Universidad de Málaga.

\section{España}

Correspondencia: Félix Moral Toranzo. Facultad de Psicología. Campus de Teatinos. CP. 29071. Málaga. España.E-mail: fmoral@uma.es

(C) Education \& Psychology I+D+i and Editorial EOS (Spain) 


\section{Resumen}

Introducción. En el ámbito educativo y social es constante el interés por investigar el tipo de consumo que los ciudadanos hacen de los diferentes medios de comunicación. Uno de los aspectos que más atención ha recibido, desde diferentes planteamientos teóricos, es el que se refiere a los efectos y la vinculación de los contenidos mediáticos con los valores personales.

Método. En una muestra de 500 estudiantes de la Universidad de Málaga (España) se han analizado sus valores sociales y los hábitos mediáticos (uso de medios de comunicación) y ciertos aspectos sociodemográficas. Los datos obtenidos a partir del cuestionario tienen por objeto profundizar en el conocimiento de estos universitarios.

Resultados. Destacan los valores sociales relativos al éxito personal, independencia, creatividad, el disfrute personal y la ayuda a los demás. Los medios más usados son Internet, televisión, prensa, radio y revistas, variando su elección en función de que los contenidos seleccionados sean informativos, formativos o de entretenimiento.

Discusión y conclusiones. Se detectan asociaciones entre el consumo de medios de comunicación y la presencia de los valores de Hedonismo, Apertura al cambio e Invidualismo. Y se ponen en evidencia diferencias significativas de los valores sociales en función del sexo, los estudios realizados, la clase social y la participación en organizaciones de voluntariado.

Palabras clave: Valores sociales, uso de los medios de comunicación, universitarios. 


\title{
Social Values and Media Habits in a Sample of University Students
}

\begin{abstract}
Introduction. The educational and social research field have shown a continued interest in knowing the media consumption habits of citizens. From different theoretical perspectives, special attention has been paid to the effects and relationships between media contents and personal values.
\end{abstract}

Method. Participants were 500 students from the University of Malaga (Spain). Social values were analyzed, measures also included media habits and socio-demographic aspects. The aim of the questionnaire was to ascertain the knowledge of these students.

Results. These results emphasize social values related to personal success, independence, creativity, personal enjoyment and helping others. The most frequently mass media used were Internet, Television, press, radio and magazines. The individual choice depended on the selected contents (that is informative, educational or entertaining).

Discussion and conclusions. The present study has shown significant relationships between the choice of mass media and the presence of Hedonism, Openness to change and individualism. Also, statistically significant differences were detected in social values related to sex, studies, social class and voluntary participation in charity organizations.

Keywords: Social values, mass media consumption habits, university students 


\section{Introducción}

Al analizar las investigaciones en torno a la influencia de los medios de comunicación, se pone de manifiesto un continuo de teorías cuyos fundamentos abarcan un extenso abanico de posibilidades. En uno de estos extremos están los modelos teóricos que defienden una influencia directa de los medios de comunicación sobre los valores y conductas de los usuarios. Aquí hay que destacar la teoría social de Bandura (1994), la teoría catártica (Noble 1973), la teoría del cultivo (Gerber y Gross, 1976) y la teoría cognitivo-asociacionista (Jo y Berkowitz 1994). En el otro lado del continuo, se sitúan las teorías que eluden una efectividad directa de los medios, donde se encuentra la teoría de usos y gratificaciones (Blumler y Katz, 1974), la teoría del efecto del entretenimiento por regulación tensional (Zillmann y Bryant, 1996), la teoría postural (Zazzo y Zazzo, 1968) y, por último, la teoría constructivista y culturalista en la que los investigadores centran su atención en estudiar los efectos de la televisión desde los conceptos de agenda setting (Severinm y Tankard, 1997), framing (Scheufele, 2004), uses and gratifications (Rubin, 1994) o disfrute (Zillmann y Bryant, 1996).

Al amparo de esta diversidad de teorías han surgido numerosas investigaciones que han tratado de poner de manifiesto el desarrollo de valores a partir de los diferentes medios de comunicación, sus contenidos o el lenguaje que utilizan. Entre esta gran variedad de trabajos sobre valores y medios de comunicación, hay que destacar los realizados por Palacios, Medrano y Cortés (2005) que, desde una perspectiva culturalista, analizan los valores implícitos y explícitos subyacentes en los contenidos televisivos, valiéndose de la creación de una escala de valores televisivos, a partir del modelo de valores de Schwartz (1992). Esto permite conocer los valores percibidos por los telespectadores en los programas de televisión que más visualizan y los valores de los propios telespectadores, siendo uno de los objetivos conocer las diferencias interindividuales de valores en función de las preferencias televisivas. En esta línea de profundizar en el conocimiento de los valores de Schwartz se enmarca la presente investigación. Pero en esta ocasión, el planteamiento del estudio se hace desde una perspectiva más amplia al contemplar a los usuarios como consumidores de los distintos medios de comunicación, y abordar el estudio de estos valores desde un óptica psicosocial que determina el comportamiento social de su conducta mediática.

Hay que reseñar que, el estudio de los valores humanos o valores en general (Rokeach, 1973; Schwartz, 1992), a lo largo de la historia, ha sido un tema de especial interés para 
disciplinas como la Educación, la Sociología o la Psicología Social. Rokeach (1973) define los valores como creencias transituacionales que se organizan de una manera jerárquica y sirven de criterio para nuestro comportamiento. Es decir, los valores tienen un carácter prescriptivo, que nos indican lo que es adecuado o inadecuado realizar. En esta línea, Schwartz (1992) desarrolla una teoría transcultural sobre los valores personales, basándose en el desarrollo y aplicación del SVS (Schwartz Values Survey) en más de 40 países. Este autor, también precisa que la motivación constituye el determinante principal en la organización de los valores, y dichos valores han de entenderse como la expresión de ciertas necesidades humanas universales, siendo susceptibles de ser agrupados en torno a diez tipos: Poder (estatus social sobre las personas y los recursos), Logros (éxito personal mediante la demostración de competencia social), Hedonismo (placer y gratificación sensual de uno mismo), Estimulación (entusiasmo, novedad y reto en la vida), Autodirección (pensamiento independiente y elección de acción, creatividad y exploración), Universalismo (comprensión, aprecio, tolerancia y protección del bienestar de todas las personas y de la naturaleza), Benevolencia (preservación e intensificación del bienestar de las personas con las que uno está en contacto personal frecuentemente). Conformidad (restricción de las acciones, inclinaciones e impulsos que pudiesen molestar o herir a otros y trasgredir normas sociales). Tradición (respeto, compromiso y aceptación de las costumbres e ideas que proporciona la cultura tradicional o la religión) y Seguridad (estabilidad de la sociedad, de las relaciones y de sí mismo). Estos valores, a su vez, se agrupan en torno a cuatro tipos de valores de orden superior, estructurados en dos dimensiones bipolares: Transcendencia (benevolencia y universalismo) versus Promoción Personal (poder, logros), y Apertura al Cambio (autodirección, estimulación) versus Conservación. Además, Schwartz, afirma que la organización de los valores puede hacerse en función de que éstos sirvan a intereses de tipo de individualista, colectivista o mixtos.

Tanto Rokeach (1973) como Schwartz (1996) tienen una visión determinista de los valores sobre el comportamiento: los valores personales son motivadores del comportamiento humano, lo que supone admitir que las conductas están más relacionadas con unos valores que con otros. Este planteamiento servirá de guía en el diseño ha esta investigación. De ahí que, entre los objetivos prioritarios de este trabajo está el analizar si la presencia de ciertos valores sociales se asocia con determinados hábitos de comportamiento mediático, posicionamiento político y social.

En lo que se refiere al ámbito general de los medios de comunicación, uno de los aspectos que más interesa es conocer cuál es la capacidad de penetración, de los diferentes me- 
dios en la población: televisión, radio, prensa, revistas o Internet. Y a partir de estos datos profundizar en los hábitos de consumo de medios de comunicación. Pero esto, también, requiere tener un conocimiento preciso de los datos cuantitativos y cualitativos, referidos estos últimos al consumo de contenidos de carácter informativo, formativo o de entretenimiento. Este conjunto de datos permite determinar si los hábitos de consumo de los diferentes medios de comunicación están proporcionados y equilibrados. En esta línea de trabajos, la Fundació Audiències de la Comunicació i la Cultura (FUNDACC), realiza de manera periódica estudios sobre los hábitos mediáticos y culturales de los ciudadanos residentes en territorios de habla catalana. Estos van a servir de referencia en la presente investigación, a la hora de diseñar el cuestionario sobre el consumo mediático.

A nivel internacional como nacional, hay un considerable número de trabajos sobre valores y medios de comunicación. En el primer caso, cabe mencionar el realizado por Puohiniemi (1995), que analizan las relaciones existentes entre valores sociales, actitudes y conductas generales de consumo. En nuestro país pueden destacarse los que se centran en el estudio del desarrollo y el aprendizaje de valores a través de la televisión (Del Río, Álvarez y Del Río, 2004; Medrano, 2005; Montero, 2006) y sobre valores y medios de comunicación (Medrano, Cortés y Palacios, 2007; Rodrigo y Medina, 2008; Rodríguez, 2009). Pero, sin duda, los trabajos que más se aproximan al objetivo propuesto en esta investigación son los desarrollados por Becker y Connor (1981), que abordan el estudio de los valores y el uso de los medios de comunicación en general. Esta investigación se llevó a cabo con una muestra compuesta por espectadores de televisión, lectores de periódicos y revistas, a los cuales les fue aplicada la escala de valores de Rockeach (1973). En sus conclusiones se indica que los valores personales influyen en la conducta individual en el uso de los medios.

\section{Objetivos e hipótesis}

Por tanto, este estudio tiene un carácter exploratorio cuyo objetivo fundamental es profundizar en el conocimiento de los jóvenes universitarios. Por un lado, se pretende dibujar el perfil de sus valores sociales, hábitos de consumo mediáticos, posicionamiento político y motivaciones sociales. Y, por otro, analizar la posible relación entre la presencia de ciertos valores y su comportamiento como usuario de medios de comunicación, y sus motivaciones políticas y sociales. En este contexto, se hipotetiza que esta muestra de jóvenes van hacer un uso preferente de Internet. Mostrarán una prevalencia de los valores de Hedonismo, Logros y Apertura al cambio. Y su consumo de medios de comunicación estará motivado mayoritaria- 
mente por el entretenimiento y asociado al valor del Hedonismo. Además, las variables sociodemográficas recogidas: sexo, edad y tipo de estudios, van a permitir conocer las posibles diferencias interindividuales.

\section{Método}

\section{Participantes}

La población general está constituida por 32.672 alumnos matriculados, durante el curso 2008/2009, en la Universidad de Málaga (España). El número total de participantes fue de 500, siendo esta muestra seleccionada de manera aleatoriamente estratificada y proporcional por titulaciones y sexo, al total del alumnado universitario. El resultado dio lugar a una muestra formada por 232 varones $(46,1 \%)$ y 271 mujeres $(53,9 \%)$, con una edad media de 20,56 años y una desviación de 2,8 años. La procedencia y distribución en los distintos centros de estudios es la siguiente (N=500): Facultad de Ciencias Económicas 58 (11,6\%), Facultad de Medicina 21 (4,2\%), Facultad de Ciencias 34 (6,8\%), Facultad de Filosofía y Letras 20 (4\%), Facultad de Derecho 31 (6,2 \%), E.T.S. de Ingeniería Informática 20 (4\%), E.T.S. de Telecomunicaciones 17 (3,4\%), E.T.S. de Ingeniería Industrial 11 (2,2\%), Facultad de Ciencias de la Comunicación 33 (6,6\%), Facultad de Ciencias de la Educación 60 (12\%), Facultad de Psicología 46 (9,2\%), Facultad de Ciencias Sociales y del Trabajo 35 (7\%), Facultad de Bellas Artes 6 (1,2\%), E.T.S. de Arquitectura 6 (1,2\%), E.U. Empresariales 19 (3,8\%), E. U. Politécnica 48 (9,6\%), Ciencias de la Salud 17 (3,4\%) y E.U. Turismo 18 $(3,6 \%)$. Su estado civil es mayoritariamente soltero/a $440(89,1 \%)$. La clase social a la que se adscriben es alta: $82(16,3 \%)$, media $366(72,8 \%)$, baja $39(7,8 \%)$ y No saben/No contestan 13 (2,6\%). Participan en Organizaciones No Gubernamentales de Desarrollo (ONGD) 153 $(30,4 \%)$, no lo hacen $339(67,4 \%)$ y No saben/No contestan $6(1,2 \%)$.

\section{Instrumentos}

Se ha utilizado un cuestionario autodescriptivo que presenta tres partes. La primera la constituye una escala sobre la dieta mediática, resultado de una la adaptación de la escala utilizada por la Fundació Audiències de la Comunicació i la Cultura (FUNDACC, 2008). Se plantean diferentes cuestiones respecto al consumo de medios de comunicación (prensa, revistas, radio, televisión e Internet): La última vez que utilizaste este medio ¿cuánto tiempo pasaste leyendo, escuchando o viendo sus contenidos? Del total del tiempo utilizado ¿qué 
porcentaje utilizaste para los contenidos informativos, formativos y de entretenimiento? La segunda parte se centra en la determinación de los valores mediante aplicación del Schwartz Values Survey o escala de valores de Schwartz (1992), adaptación española (Ros y Grand, 1991), que consta de 56 ítems, con un formato de respuesta en intervalos de 0 a 7 (desde nada importante en mi vida a de extrema importancia), cuya fiabilidad queda patente al presentar un alfa de Cronbach de 0,86. Y la última parte se refiere a diferentes aspectos sociodemográficos: sexo, edad, estado civil, tipo de estudios, clase social y participación en ONGD.

\section{Procedimiento}

Los entrevistadores, que previamente habían sido entrenados para el desarrollo de los cuestionarios, se desplazaron por las diferentes escuelas y facultades de la Universidad de Málaga. En cada uno de estos centros se había establecido, de antemano, el número y el sexo de los participantes que formarían la parte proporcional del total de la muestra. Los alumnos/as que accedían a contestar al cuestionario de la investigación lo hacían de manera voluntaria. Y una vez recogidos los cuestionarios, se comprobó su correcta cumplimentación y adecuación a las instrucciones dadas, para posteriormente proceder a la grabación de los datos en el programa estadístico SPSS (15.0) y acometer su análisis.

\section{Análisis de datos}

Los datos para este estudio han sido recogidos a través de diferentes escalas multirespuesta y sometidos a un análisis multivariante. Por tanto, el método empleado es de tipo descriptivo-comparativo. Se hacen análisis descriptivos (frecuencia, media aritmética y desviación típica) de los valores sociales y del uso de los medios de comunicación, y comparaciones entre estas variables mediante las pruebas de correlación (Spearman y Pearson) y varianza intergrupal (t de Student y F de Fisher) en función de ciertas características sociodemográficas.

\section{Resultados}

Los hábitos mediáticos de estos jóvenes universitarios se caracterizan por un uso mayoritario de Internet, seguido de la televisión, prensa, radio y revistas. Donde pueden observarse diferentes motivaciones según el medio de comunicación. En prensa, la principal moti- 
vación son los contenidos informativos, mientras que en revistas, radio y televisión lo son los contenidos de entretenimiento (véase tabla 1).

Tabla 1. Uso de medios de comunicación

\begin{tabular}{lcccccccc}
\hline & Uso diario & \multicolumn{1}{c}{ Motivaciones de uso } \\
& & \multicolumn{9}{c}{ Informativos } & \multicolumn{2}{c}{ Formativos } & \multicolumn{2}{c}{ Entretenimiento } \\
& $\mathrm{N}$ & $\%$ & $M$ & $D T$ & $M$ & $D T$ & $M$ & $D T$ \\
\hline Prensa & 242 & 48,1 & 40,61 & $(19,29)$ & 23,34 & $(14,91)$ & 35,96 & $(21,60)$ \\
Revistas & 45 & 11,3 & 30,36 & $(21,94)$ & 26,18 & $(20,36)$ & 43,17 & $(28,84)$ \\
(mensual) & & & & & & & & \\
Radio & 181 & 36 & 17,09 & $(17,96)$ & 11,75 & $(14,35)$ & 70,96 & $(25,61)$ \\
Televisión & 279 & 71,9 & 28,04 & $(15,17)$ & 18,28 & $(15,17)$ & 53,63 & $(21)$ \\
Internet & 434 & 86,3 & & & & & & \\
\hline
\end{tabular}

En lo que respecta a los valores sociales, a partir de la escala de valores de Schwartz (1992), el primer nivel de valores presenta el siguiente orden de frecuencia: Logros, Autodirección, Hedonismo, Universalismo, Benevolencia, Seguridad, Conformidad, Estimulación, Tradición y Poder. Y para el segundo nivel: Apertura al Cambio y Conservación, Transcendencia y Promoción Personal, Mixto, Individualismo y Colectivismo. En la tabla 2 se exponen las medias y desviaciones típicas obtenidos en cada uno de estos valores. 
Tabla 2. Valores sociales: medias y desviaciones típicas

\begin{tabular}{lcc}
\hline Valores & $M$ & $D T$ \\
\hline Primer nivel & & \\
\hline Universalismo & 4,64 & $(0,81)$ \\
Benevolencia & 4,64 & $(0,67)$ \\
Tradición & 3,51 & $(0,82)$ \\
Conformidad & 4,42 & $(1,00)$ \\
Seguridad & 4,57 & $(0,79)$ \\
Poder & 3,10 & $(1,13)$ \\
Logros & 5,54 & $(0,93)$ \\
Hedonismo & 4,96 & $(1,09)$ \\
Estimulación & 4,30 & $(1,16)$ \\
Autodirección & 4,98 & $(0,69)$ \\
Segundo nivel & & \\
\hline Transcedencia $v s$ & 4,64 & $(0,65)$ \\
Promoción personal & 4,32 & $(0,86)$ \\
Apertura al cambio $v s$ & 4,75 & $(0,76)$ \\
Conservación & 4,17 & $(0,70)$ \\
Colectivismo & 4,19 & $(0,69)$ \\
Individualismo & 4,58 & $(0,67)$ \\
Mixto & 4,61 & $(0,65)$ \\
\hline
\end{tabular}

En la búsqueda de asociaciones significativas entre valores sociales y el consumo de medios, el análisis correlacional de Spearman ponen en evidencia las siguientes: Hedonismo $(r=.095, p<.05)$, Apertura al Cambio $(r=.107, p<.05)$ e Individualismo $(r=.091, p<.05)$. Y al profundizar en el análisis entre los valores sociales y las motivaciones en el uso de los medios (informativos, formativos y entretenimiento), mediante la correlación de Pearson, en la tabla 3, se muestran asociaciones significativas entre: las preferencias por los contenidos informativos y los valores sociales de Universalismo, Transcendencia y Mixto $(r x y=.105, p<.05)$. La motivación de uso de los medios de tipo formativo con el Universalismo, Seguridad, Poder y Transcendencia. Y en la motivación de uso del entretenimiento en los medios y el Universalismo, Trascendencia y Apertura. 
Tabla 3. Correlacciones entre los valores sociales y usos de los medios de comunicación

\begin{tabular}{|c|c|c|c|}
\hline & $\begin{array}{l}\text { Uso informativo de los } \\
\text { Medios de comunicación }\end{array}$ & $\begin{array}{c}\text { Uso formativo de los } \\
\text { medios de comunicación }\end{array}$ & $\begin{array}{c}\text { Uso de entretenimiento } \\
\text { de los medios de comunicación }\end{array}$ \\
\hline \multicolumn{4}{|l|}{$\overline{\text { Universalismo }}$} \\
\hline Correlación de Pearson &, 157 & 143 &,- 205 \\
\hline Significación (bilateral) &, $000 * * *$ & $001 * *$ &, $000 * * *$ \\
\hline \multicolumn{4}{|l|}{ Benevolencia } \\
\hline Correlación de Pearson & ,617 & 033 &,- 082 \\
\hline Significación (bilateral) &, 466 &, 052 & 069 \\
\hline \multicolumn{4}{|l|}{ Tradición } \\
\hline Correlación de Pearson & 040 & 071 &,- 082 \\
\hline Significación (bilateral) &, 370 & 113 & ,068 \\
\hline \multicolumn{4}{|l|}{ Conformidad } \\
\hline Correlación de Pearson & 079 &,- 073 &,- 007 \\
\hline Significación (bilateral) & 077 &, 104 & 884 \\
\hline \multicolumn{4}{|l|}{ Seguridad } \\
\hline Correlación de Pearson &, 012 &,- 119 & ,066 \\
\hline Significación (bilateral) &, 786 &, $008 * *$ & 139 \\
\hline \multicolumn{4}{|l|}{ Poder } \\
\hline Correlación de Pearson & 010 &,- 103 & 061 \\
\hline Significación (bilateral) & 824 &, $022 * *$ & 176 \\
\hline \multicolumn{4}{|l|}{ Logros } \\
\hline Correlación de Pearson &, 062 &,- 068 & ,004 \\
\hline Significación (bilateral) &, 169 & 130 & ,922 \\
\hline \multicolumn{4}{|l|}{ Hedonismo } \\
\hline Correlación de Pearson & 007 &,- 026 & ,006 \\
\hline Significación (bilateral) & 869 &, 555 & 898 \\
\hline \multicolumn{4}{|l|}{ Estimulación } \\
\hline Correlación de Pearson & 038 & 056 &,- 065 \\
\hline Significación (bilateral) & ,396 &, 214 &, 149 \\
\hline \multicolumn{4}{|l|}{ Autodirección } \\
\hline Correlación de Pearson &, 147 & ,073 &,- 149 \\
\hline Significación (bilateral) & $001 * *$ & 103 &, $001 * *$ \\
\hline \multicolumn{4}{|l|}{ Promoción personal } \\
\hline Correlación de Pearson &, 038 &,- 096 &, 037 \\
\hline Significación (bilateral) & ,393 & 034 & ,406 \\
\hline \multicolumn{4}{|l|}{ Trascendencia } \\
\hline Correlación de Pearson &, 109 &, 137 &,- 166 \\
\hline Significación (bilateral) & $016^{* *}$ &, $002 * *$ &, $000 * * *$ \\
\hline \multicolumn{4}{|l|}{ Apertura al cambio } \\
\hline Correlación de Pearson & 075 & 046 &,- 085 \\
\hline Significación (bilateral) & ,098 &, 312 &, 060 \\
\hline \multicolumn{4}{|l|}{ Conservación } \\
\hline Correlación de Pearson & 048 &,- 046 &,- 007 \\
\hline Significación (bilateral) & 292 &, 307 &, 885 \\
\hline \multicolumn{4}{|l|}{ Colectivismo } \\
\hline Correlación de Pearson &, 058 & 021 &,- 056 \\
\hline Significación (bilateral) & 200 & 645 & 215 \\
\hline \multicolumn{4}{|l|}{ Individualismo } \\
\hline Correlación de Pearson & 067 &,- 019 &,- 036 \\
\hline Significación (bilateral) & 139 & 673 &, 428 \\
\hline \multicolumn{4}{|l|}{ Mixto } \\
\hline Correlación de Pearson &, 105 & 016 &,- 087 \\
\hline Significación (bilateral) & $019 * *$ &, 720 & 052 \\
\hline
\end{tabular}


Otra de las inquietudes de este trabajo es la de comparar los valores sociales, en función del sexo. El resultado de esta comparación, mediante la prueba $t$ de Student (tabla 4), muestra diferencias significativas en los casos de Universalismo, Benevolencia, Tradición, Seguridad, Trascendencia, Conservación, Colectivismo y Mixto, siendo en todos ellos la media de estos valores sociales superior en el sexo femenino

Tabla 4. Análisis diferencial de los valores sociales según el sexo

\begin{tabular}{|c|c|c|c|c|c|c|}
\hline \multirow[b]{2}{*}{ Valores sociales } & \multicolumn{2}{|c|}{ Varón } & \multicolumn{2}{|c|}{ Mujer } & \multirow[b]{2}{*}{$T$} & \multirow[b]{2}{*}{ Sig. } \\
\hline & $M$ & $D T$ & $M$ & $D T$ & & \\
\hline Universalismo & 4,52 & $(0,85)$ & 4,74 & $(0,76)$ & $-3,013$ & $.003 * *$ \\
\hline Benevolencia & 4,54 & $(0,64)$ & 4,73 & $(0,69)$ & $-3,013$ & $.003 * *$ \\
\hline Tradición & 3,41 & $(0,81)$ & 3,60 & $(0,82)$ & $-2,580$ & $.010^{*}$ \\
\hline Conformidad & 4,36 & $(1,00)$ & 4,48 & $(1,00)$ & $-1,322$ & .187 \\
\hline Seguridad & 4,48 & $(0,80)$ & 4,66 & $(0,78)$ & $-2,564$ & $.011^{*}$ \\
\hline Poder & 3,16 & $(1,15)$ & 3,05 & $(1,12)$ & 1,087 & .277 \\
\hline Logros & 5,60 & $(0,91)$ & 5,48 & $(0,95)$ & 1,397 & .163 \\
\hline Hedonismo & 4,98 & $(1,14)$ & 4,93 & $(1,04)$ &, 514 & .608 \\
\hline Estimulación & 4,35 & $(1,18)$ & 4,26 & $(1,15)$ & 800 & .424 \\
\hline Autodirección & 5,02 & $(0,67)$ & 4,95 & $(0,71)$ & 1,228 & .220 \\
\hline Promoción personal & 4,38 & $(0,85)$ & 4,27 & $(0,87)$ & 1,402 & .162 \\
\hline Trascendencia & 4,53 & $(0,64)$ & 4,73 & $(0,65)$ & $-3,383$ & $.001 * *$ \\
\hline Apertura al cambio & 4,79 & $(0,76)$ & 4,71 & $(0,75)$ & 1,125 & .261 \\
\hline Conservación & 4,08 & $(0,68)$ & 4,24 & $(0,72)$ & $-2,526$ & $.012 *$ \\
\hline Colectivismo & 4,10 & $(0,66)$ & 4,26 & $(0,71)$ & $-2,561$ & $.011^{*}$ \\
\hline Individualismo & 4,63 & $(0,66)$ & 4,54 & $(0,69)$ & 1,527 & .127 \\
\hline Mixto & 4,50 & $(0,64)$ & 4,70 & $(0,64)$ & $-3,454$ & $.001 * *$ \\
\hline
\end{tabular}

En relación a la participación social de los universitarios en ONGD, del total de la muestra, $339(68,8 \%)$ responden negativamente, $153(31,2 \%)$ afirmativamente y 8 No saben/No constentan. En base a esta distinción, se realiza una comparación de sus valores sociales, mediante la prueba $t$ Student, cuyos resultados se exponen en la tabla 5 , donde se aprecian diferencias entre ambos grupos. En el grupo de los que participan en ONGD destacan los valores de Universalismo, Benevolencia y Trascendencia, mientras que los que no participan en ONGD lo hacen en Poder, Hedonismo, Promoción Personal e Individualidad. 
Tabla 5. Análisis diferencial de los valores sociales según la participación en ONGD

\begin{tabular}{lcccccc}
\hline & \multicolumn{7}{c}{ Participan en } & \multicolumn{7}{c}{ No participan } \\
& \multicolumn{2}{c}{ ONGD } & \multicolumn{2}{c}{ en ONGD } & \\
Valores sociales & $M$ & $D T$ & $M$ & $D T$ & $T$ & Sig. \\
\hline Universalismo & 4,77 & $(, 76)$ & 4,58 & $(, 82)$ & 2,349 & $.019 *$ \\
Benevolencia & 4,80 & $(, 65)$ & 4,58 & $(, 67)$ & 3,334 & $.001 * *$ \\
Tradición & 3,47 & $(, 83)$ & 3,54 & $(, 82)$ &,- 909 & .364 \\
Conformidad & 4,37 & $(1)$ & 4,45 & $(, 99)$ &,- 846 & .398 \\
Seguridad & 4,50 & $(, 79)$ & 4,62 & $(, 79)$ & $-1,478$ & .140 \\
Poder & 2,82 & $(1,13)$ & 3,24 & $(1,11)$ & $-3,874$ & $.000 * * *$ \\
Logros & 5,46 & $(, 94)$ & 5,57 & $(, 93)$ & $-1,290$ & .198 \\
Hedonismo & 4,78 & $(1,19)$ & 5,03 & $(1,04)$ & $-2,332$ & $.020 *$ \\
Estimulación & 4,28 & $(1,18)$ & 4,30 & $(1,17)$ &,- 171 & .864 \\
Autodirección & 5,02 & $(, 69)$ & 4,96 & $(, 70)$ &, 878 & .380 \\
Promoción personal & 4,14 & $(, 86)$ & 4,41 & $(, 85)$ & $-3,201$ & $.001 * *$ \\
Trascendencia & 4,78 & $(, 59)$ & 4,58 & $(, 67)$ & 3,155 & $.002 * *$ \\
Apertura al cambio & 4,71 & $(, 78)$ & 4,76 & $(, 75)$ &,- 757 & .449 \\
Conservación & 4,10 & $(, 71)$ & 4,20 & $(, 70)$ & $-1,348$ & .178 \\
Colectivismo & 4,20 & $(, 68)$ & 4,19 & $(, 69)$ &, 221 & .825 \\
Individualismo & 4,48 & $(, 68)$ & 4,62 & $(, 68)$ & $-2,143$ & $.033 *$ \\
Mixto & 4,64 & $(, 62)$ & 4,60 & $(, 66)$ &, 638 & .524 \\
\hline$* p<.05 ; * * p<.01 ; p<.001$ & & & & & & \\
\hline & & & & & &
\end{tabular}

En lo que respecta a la clase social, y con objeto de conocer la existencia de diferencias intergrupales respecto a sus valores sociales, se realiza una Anova, resultando significativa la diferencia en Universalismo $[\mathrm{F}(2,480)=5,034 ; p=.007]$. Y realizada, a posteriori, la prueba de Bonferroni, las diferencias se muestran significativas al comparar las medias de la clase social alta con la media $=-, 27 ; p<.05$ y de la clase social alta con la baja $=-, 43 ; p<.05$.

También se ha descrito la distribución de estos universitarios en función de los estudios que realizan. En base a estos grupos, y para determinar las posibles diferencias entre sus valores sociales, se realiza una Anova, que muestra diferencias significativas entre los siguientes valores sociales: Universalismo $[\mathrm{F}(17,493)=3.357 ; \quad p=.000]$, Poder $[\mathrm{F}(17,494)=2.870 ; p=.000$, Promoción Personal $[\mathrm{F}(17,491)=1.727 ; p=.035]$, Trascendencia $[\mathrm{F}(17,486)=2.917 ; p=.000]$ y Mixto $[\mathrm{F}(17,491)=2.274 ; p=.003]$. Y al ser analizadas las diferencias de medias, posteriormente con la prueba de Bonferroni, se muestran significativas en 
los siguientes casos: en Universalismo al comparar las medias de la Facultad de Económicas con Facultad de Ciencias= -,67; $p<.01$; las de la Facultad de Bellas Artes con las facultades o escuelas de Derecho= $=1,50, p<.01$, Informática $=1,60, p<.01$, Industriales $=1,58, p<0.05 \mathrm{e}$ Ingeniería Técnica $=1,51, p<.05$. En el Poder al comparar las medias de Facultad de Económicas con la Facultad de Ciencias de la Comunicación= 1,06, $p<.01$, y de la Diplomatura de Empresariales con las facultades y escuelas de Ciencias $=1,14, p<.05$, Comunicación=1,55, $p<.01$, Arquitectura $=2,05, p<.01$ y Ciencias Salud=1,37, $p<.05$. En el valor de Promoción Personal al comparar las medias de la Diplomatura de Empresariales y la Facultad de Ciencias comunicación=0,98; $p<.05$. En la Trascendencia al comparar las medias de la Facultad de Económicas con la facultad de Ciencias Comunicación= -0,51, p<.05, y la Facultad de Bellas Artes con las facultades y escuelas de Económicas $=1,21, p<.05$, Derecho $=1,13$, $p<.05$, Informática $=1,23, \mathrm{p}<.05$ e Ingeniería Técnica $=1,19, p<.05$. Y por último el valor social Mixto al comparar las medias de Bellas Artes con las facultades y escuelas de Económicas $=1,14, p<0.05$, Derecho $=1,08, p<.05$, Ingeniería Informática $=1,20, p<.05$, Industriales $=1,21, p<.05$, Comunicación= 1,15, $p<.05$ e Ingenierías Técnicas=1,21, $p<.01$.

\section{Discusión y conclusiones}

Este trabajo ha sido realizado con una muestra de estudiantes de la Universidad de Málaga, cuyo número se ha elegido de manera aleatoria y proporcional a los diferentes estudios y el sexo de estos universitarios, pero dada su cuantía no puede considerarse representativo de toda la población, ni sus resultados generalizables, sino más bien con un carácter exploratorio y referencial.

Entre los resultados obtenidos hay que resaltar que, en los valores sociales de estos jóvenes universitarios destacan, por un lado, las motivaciones asociadas al éxito personal (Logros), la independencia, creatividad y exploración (Autodirección), el placer y el disfrute personal (Hedonismo), la ayuda a los demás y la honestidad (Benevolencia), y, por otro, las relativas a la Trascendencia: tolerancia, igualdad social y honestidad, Apertura al cambio: creatividad y libertad; y Mixto: la tolerancia, justicia social, seguridad y orden social. Este contraste de valores presentes en los jóvenes puede ser sintomático de la fragmentación de valores que caracteriza a la juventud postmodernista (Bernal, 2009; Elzo, 1999), acorde con una sociedad occidentalizada, donde las convicciones están muy dispersas. 
Sus hábitos mediáticos están marcados por el uso preferente de Internet, al que le sigue la televisión, y posteriormente, la prensa, radio y revistas. La elección de uno u otro medio, en función de sus contenidos, revela una preferencia por los contenidos informativos y de entretenimiento y, en ningún caso, los contenidos formativos marcan un uso destacado. Este reparto revela un consumo cualitativo desproporcionado; es decir, que su dieta mediática es desequilibrada (Nosty, 2005). El desajuste observado coincide, en la actualidad, con una creciente oferta de contenidos de entretenimiento, donde destacan los valores hedonistas, materialistas e individualistas (Del Río et al., 2004). Lo cual se ve corroborado, en este estudio, con una asociación positiva entre el consumo de medios de comunicación y la presencia destacada de valores como Hedonismo, Apertura al cambio e Individualismo. Esta sintonía de valores en las programaciones de los medios y los valores de los usuarios coincide con los planteamientos de otros trabajos de Palacios, Medrano y Cortés (2005), en ámbitos universitarios, sobre preferencias televisivas y la escala de valores de Schwartz. Y esta vinculación entre valores y contenidos televisivos, a juicio de diferentes autores (Buckingham, 2003; Gentile y Walsh, 2002; Morley, 2006), deber ser aprovechada para reforzar determinadas conductas y afianzar ciertos valores como la Autodirección, Benevolencia y Logros. Además, se aprecian interesantes diferencias en los valores de los usuarios en la selección de los contenidos de los medios: la elección de contenidos informativos y formativos se asocia con la presencia de los valores de Universalismo y Trascendencia y, en cambio, la ausencia de éstos se asocia con la elección de contenidos de entretenimiento. Estas relaciones pueden estar apuntando hacia un determinismo en la presencia de ciertos valores y las preferencias en los medios. En línea con los recientes trabajos desarrollados por Doran (2009), a partir de la escala de valores de Schwartz, que han puesto de manifiesto la prevalencia de ciertos valores personales en los consumidores estadounidenses de productos de comercio justo. Pero la confirmación de esta previsión requiere la realización de estudios futuros que contemplen el uso de metodologías causales y en la búsqueda de la influencia de estos valores en otros ámbitos sociales.

Uno de los intereses de este estudio ha sido la búsqueda, en esta muestra de universitarios, de diferencias en función de las variables sociodemográficas analizadas. Así, de los 18 centros de estudios de la Universidad de Málaga (España), tan sólo se manifiestan de manera distintiva los alumnos de Bellas Artes, Económicas y Empresariales. En el caso de Bellas Artes, los alumnos se caracterizan por la tolerancia, comprensión y búsqueda del bienestar en quienes les rodean. Y, en cambio, en los que cursan Económicas y Empresariales predominan la búsqueda del éxito y de estatus social. Lo cual puede ser interesante a la hora poder expli- 
car las motivaciones que guían la elección de estos estudios. También resulta revelador comprobar que las mujeres difieren con los varones, en casi la mitad de los valores analizados, y en todos estos casos los superan. Entre estos valores figuran los de Benevolencia y Universalismo, que han sido designados en anteriores trabajos como característicos del sexo femenino (Schwartz y Rubel, 2005). Lo que, sin duda, apunta al sexo como una variable determinante en la presencia de ciertos valores.

Otras de las variables estudiadas para describir a esta muestra de universitarios malagueños han sido la clase social y la participación social. Las cuales han sido analizadas, en trabajos anteriores de Schwartz (2006), al abordar la influencia de los valores en los procesos de democratización social y gobierno. En lo que se refiere a la clase social, se aprecia que el Universalismo se manifiesta de manera más destacada en las clases sociales media y baja. Respecto a la participación, se pone de manifiesto que la mayoría no colaboran con ONGD, presentando este grupo un dominio de los valores de Poder, Hedonismo e Individualidad, mientras que aquellos que sí colaboran se caracterizan por el Universalismo, Benevolencia, Promoción Personal y Transcendencia. Estos perfiles, en ambos casos, se muestran acordes los valores con las conductas manifiestas.

Así, desde un planteamiento culturalista se hace evidente la existencia de valores en los medios de comunicación y sus usuarios (Medrano, Cortés y Palacios, 2007). Pero los medios de comunicación sólo representan una parte en los procesos de influencia y en la conformación de los valores de la persona, donde el contexto social y educativo ocupa un papel trascendental. De ahí, que la escala de valores de Schwartz sea un instrumento adecuado para detectar, por un lado, los valores implícitos y explícitos que subyacen en los contenidos de los medios de comunicación y, por otro, dibujar un perfil de valores en los usuarios. En este caso, el de una muestra de jóvenes universitarios, que ha permitido explorar y profundizar su conocimiento personal y comportamiento mediático, desde la perspectiva del modelo de valores de Schwartz, que, de manera modesta, se suma a las numerosas investigaciones internacionales que se han marcado como objetivo el estudio de la influencia de los valores sociales en diferentes aspectos individuales y sociales de las personas, entre las que pueden citarse: la conducta moral (Bond y Chi, 1997), las actitudes y conductas proambientales (Nordlund y Garvill, 2002), la confianza en las instituciones (Devos, Spini y Schwartz, 2002) o el comercio internacional y la distancia intercultural (Ng, Lee y Soutar, 2007; Schwartz, 2007). 
Los resultados obtenidos confirman algunas de las previsiones hipotetizadas, en lo que se refiere al uso preferente de Internet, el lugar destacado que ocupan los valores de Hedonismo, Logros y Apertura al cambio y el uso de los medios de comunicación, motivado mayoritariamente por el entretenimiento y la información. Además, se revelan aspectos diferenciales en los valores que poseen en función del sexo, los estudios cursados o la participación en ONGD.

Todo ello, anima a la realización de otros estudios con muestras representativas que permitan la generalización de los resultados, que pueda abarcar, no sólo al ámbito provincial, sino, también, al autonómico o nacional. Además, sería interesante incluir en futuras investigaciones otras fuentes de influencia en los valores sociales y hábitos mediáticos, como pueden ser los diferentes hábitos culturales o las múltiples estrategias educativas. 


\section{Referencias}

Bandura, A. (1994). Social cognitive theory of mass communication. En J. Bryant y D. Zillman (Eds.), Media effects: advances in theory and research (pp. 61-90). Hillsdale: NJ Erlbaum.

Becker, B. y Connor, P. (1981). Personal values of the Heavy User of Mass Media. Journal of Advertsing Researhc, 5, 37-44.

Bernal, A.I. (2009). Los nuevos medios de comunicación y los jóvenes. Madrid, Euroeditions, Fundación Europea para la Sociedad de la Información y la Administración Electrónica.

Blumer, J. Katz, E. (1974). The uses of mass comunications. Beverly Hills: CA-Sage.

Bond, M. H. y Chi, V. M. (1997) Values and moral behaviour in mainland China. Psychologia, 40 (4), 251-264.

Devos, T., Spini, D. y Schwartz, S.H. (2002).Conflicts Among Human Values and Trust in Institutions. British Journal of Social Psychology, 41, 481-494.

Buckingham, D. (2003) Media education. Literacy, Learning and Contemporary Culture. Cambridge, Polity Press.

Del Río, P., Álvarez, A. y Del Río, M. (2004). Pigmalión. Informe sobre el impacto de la televisión en la infancia. Madrid: Fundación Infancia y Aprendizaje.

Doran, Caroline. J. (2009). The role of personal values in fair trade consumption: An investigation of ethical consumption practices. Journal of Busines Ethics, 84, 549-563.

Elzo, J. (1999). Jóvenes españoles 99. Madrid. Fundación Santa María.

Fundació Audiències de la Comunicació i la Cultura FUNDACC (2008). El barómetro de la comunicación y la cultura. Obtenido el 30/06/2008 en http://www.fundacc.org.

Gentil, A. D. y Walsch, D. A. (2002) A normative study of family media habits, Applied Developmental Psychology, 23, 157-178.

Gerber, G. y Gross, L. (1976). Living with television: the violence profile. Journal Comunication, 26, 173-179.

Jo, E. y Berkowitz, L. (1994): A priming effect analysis on media in.uences: an update». En J. Bryant y D. Zillman (Eds.), Media effects: advances in theory and research (pp.4360). Hillsdale: NJ Erlbaum.

Medrano, C. (2005). ¿Se puede favorecer el aprendizaje de valores a través de las narraciones televisivas? Revista de Educación, 338, 245-270.

Medrano, C., Cortés, M. P. y Palacios, S. (2007). La televisión y el desarrollo de valores. Revista de Educación, 342, 307-328. 
Montero, Y. (2006). Televisión, valores y adolescentes. Barcelona: Gedisa.

Morley, D. (2006) Unanswered questions in audience research. The Communication Review, $9,101-121$.

Ng, S.I., Lee, J.A., Soutar, G.N. 2007, 'Are Hofstede's and Schwartz's value frameworks congruent?', International Marketing Review, 24, 2, pp. 164-180.

Nordlund, A. M., \& Garvill, J. (2002).Value structures behind pro-environmental behavior. Environment and Behavior, 34, 740-756.

Noble, G. (1973). Effects, of different forms of filmed agresion on chlidren's constructive and destructive play. Journal of Personality and Social Psychology, 26, 54-59.

Díaz Nosty, B. (2005). El déficit mediático. Barcelona: Bosch.

Palacios, S., Medrano, C. y Cortés, P. A. (2005). Valores y preferencias televisivas una experiencia universitaria. Comunicar, 25, 290.

Puohiniemi, M. (1995). Values, consumer attitudes and behaviour. An application of Schwartz's value theory to the analysis of consumer behaviour and attitudes in two national samples. Helsinki: University of Helsinki, Department of Social Psychology.

Rodrigo, M. y Medina, P. (2008). Juventud y Comunicación: el impacto de los medios de comunicación en la transmisión de valores interculturales. Revista de Estudios de Juventud, $80,151-167$.

Rodríguez, J. L. (2009). Los medios de comunicación y la formación en valores, actitudes y hábitos de convivencia. Zaragoza: Techtraining Multiservice, S.L.

Rokeach, M. (1973). The nature of human values. Nueva York: The Free Press.

Ros, M. y Grad, H. (1991): El significado del valor trabajo como relacionado a la experiencia ocupacional: una comparación de profesores de EGB y estudiantes del CAP. Revista de Psicología Social, 6, 2, pp. 181-208.

Rubin, A.M. (1994). Media Uses and Effects: A Uses and Gratifications Perspective. En J. Bryant y D. Zillman (Eds.), Media effects: advances in theory and research (pp.417436). Hillsdale: NJ, Erlbaum.

Scheufele, B. (2004). Framing-effects approach: A theoretical and methodological critique. Communications, 29, 401-428.

Schwartz, S.H. (1992). Universals in the content and structure of values: Theoretical advances and empirical tests in 20 countries. Advances in Experimental Social Psychology, 25, 1-65.

Schwartz, S. H. (2006). A Theory of Cultural Value Orientations: Explication and Applications. Comparative Sociology 5, 137-182. 
Schwartz, S. H. ( 2007). Universalism Values and the Inclusiveness of Our Moral Universe. Journal of Cross-Cultural Psychology 38, 711-728.

Schwartz, S. H. y Rubel, T. (2005). Sex differences in value priorities: Cross-cultural and multimethod studies. Journal of Personality and Social Psychology, 89, 1010-1028.

Severinm, W.J. y Tankard, J.W. (1997). Communications Theories: Origins, Methods, and Uses in the Mass Media. New York: Longman Publishers.

Zazzo, R. y Zazzo, B. (1962). Les films pour les enfants de 7 à 12 ans. En R. Zazzo (Edt.), Conduites et consciente (pp.163-180). Neuchàtel: Delachaux-Niestlé.

Zillmann, D. y Bryant, J. (1996). El entretenimiento como efecto de los medios. En J. Bryant y D. Zillmann (Eds.) Los efectos de los medios de comunicación. Investigaciones y teorías (pp.583-616). Barcelona: Paidós. 